\title{
Hauteur de Faltings et hauteur de Néron-Tate du diviseur thêta
}

\author{
Pascal Autissier
}

\begin{abstract}
In this paper we prove a formula relating the Faltings height of an abelian variety $A$ over $\overline{\mathbb{Q}}$ and the Néron-Tate height of a theta divisor on $A$.
\end{abstract}

\section{RÉSUMÉ}

On montre dans ce texte une formule reliant la hauteur de Faltings d'une variété abélienne $A$ sur $\overline{\mathbb{Q}}$ et la hauteur de Néron-Tate d'un diviseur thêta sur $A$.

\section{Introduction}

On étudie dans ce travail le lien entre la hauteur de Faltings d'une variété abélienne $A$ et la hauteur de Néron-Tate d'un diviseur thêta sur $A$. Introduisons d'abord quelques notations :

Dans toute la suite, on note $\kappa_{0}$ la constante $\kappa_{0}=\ln (\pi \sqrt{2})$. Soit $g$ un entier $\geqslant 1$. On désigne par $\mathcal{A}_{g}$ le schéma de modules (grossier) des schémas abéliens de dimension relative $g$ et principalement polarisés.

Soit $K \subset \overline{\mathbb{Q}}$ un corps de nombres de degré $N$. Notons $G_{K}$ l'ensemble des plongements $\sigma: K \hookrightarrow \mathbb{C}$. Pour tout idéal maximal $b$ de $O_{K}$, on pose $\mathrm{N}_{b}=\#\left(O_{K} / b\right)$.

Soit $A$ une variété abélienne de dimension $g$ sur $K$. On désigne par $\hat{\mathrm{h}}\left(A_{\overline{\mathbb{Q}}}\right)$ la hauteur de Faltings (stable) de $A_{\overline{\mathbb{Q}}}$. Lorsque $L$ est un faisceau inversible symétrique et ample sur $A$, on note $\mathrm{h}_{L}^{\prime}$ la fonction hauteur de Néron-Tate sur $A$.

Observons que $\mathrm{h}_{L}^{\prime}$ et $\hat{\mathrm{h}}$ ne sont pas de même nature a priori $: \mathrm{h}_{L}^{\prime}$ est une hauteur sur $A$, tandis que $\hat{\mathrm{h}}$ est une hauteur sur $\mathcal{A}_{g}(\overline{\mathbb{Q}})$. On montre cependant la relation suivante :

ThÉorème (Théorème 3.1). On suppose que $A$ a potentiellement bonne réduction partout. Soit $\Theta$ un diviseur effectif symétrique et ample sur $A$, définissant une polarisation principale $\lambda$ de $A$. En posant $L=\mathcal{O}_{A}(\Theta)$, on a alors l'égalité suivante :

$$
\hat{\mathrm{h}}\left(A_{\overline{\mathbb{Q}}}\right)=2 g \mathrm{~h}_{L}^{\prime}(\Theta)-\kappa_{0} g+\frac{2}{N} \sum_{\sigma \in G_{K}} I\left(A_{\sigma} ; \lambda_{\sigma}\right) .
$$

Dans cet énoncé, $I$ est une fonction continue explicite $\mathcal{A}_{g}(\mathbb{C}) \rightarrow \mathbb{R}_{+}(\mathrm{cf}$. $\S 2.1)$, que l'on peut voir comme une hauteur locale en la place archimédienne. On prouve au $\S 4$ que $I(A ; \lambda)$ tend vers $+\infty$ lorsque $(A ; \lambda)$ tend vers l'infini dans $\mathcal{A}_{g}(\mathbb{C})$.

La démonstration du théorème 3.1 repose sur la 'formule clef' de Moret-Bailly (cf. [Mor85, § 8.1] et [Mor90]) et sur les propriétés élémentaires des hauteurs d'Arakelov (cf. [BGS94]).

Received 5 December 2005, accepted in final form 10 May 2006.

2000 Mathematics Subject Classification 14G40, 11G10, 14K15, $11 \mathrm{G} 50$.

Keywords: height, abelian variety, Arakelov theory, theta-function.

This journal is (C) Foundation Compositio Mathematica 2006. 


\section{P. Autissier}

Ce résultat suggère que $\hat{\mathrm{h}}\left(A_{\overline{\mathbb{Q}}}\right)-2 g \mathrm{~h}_{L}^{\prime}(\Theta)$ admet peut-être une décomposition en termes locaux, même sans hypothèse de bonne réduction :

Question. Soit $A$ une variété abélienne semi-stable de dimension $g$ sur $K$. Soit $\Theta$ un diviseur effectif symétrique et ample sur $A$, définissant une polarisation principale $\lambda$ de $A$. On pose $L=\mathcal{O}_{A}(\Theta)$. A-t-on une formule du type

$$
\hat{\mathrm{h}}\left(A_{\overline{\mathbb{Q}}}\right)=2 g \mathrm{~h}_{L}^{\prime}(\Theta)+\frac{1}{N} \sum_{b} \alpha_{b} \ln \mathrm{N}_{b}+\frac{2}{N} \sum_{\sigma} I\left(A_{\sigma} ; \lambda_{\sigma}\right)-\kappa_{0} g,
$$

où pour tout idéal maximal $b$ de $O_{K}, \alpha_{b}$ est un rationnel positif ou nul calculable en fonction de la réduction de $A$ modulo $b$ (et $\alpha_{b}=0$ si $A$ a bonne réduction en $b$ )?

ThÉorème 1.1. La réponse est positive si $g \leqslant 2$, et plus généralement si $\left(A_{\overline{\mathbb{Q}}} ; \lambda_{\overline{\mathbb{Q}}}\right)$ est un produit de courbes elliptiques et de surfaces abéliennes principalement polarisées.

Pour démontrer ce théorème, il suffit, par additivité, de considérer les deux cas suivants :

- A est une courbe elliptique; le résultat est alors connu (cf. théorème 7 de Faltings [Fal84]) avec les précisions suivantes: on a $\mathrm{h}_{L}^{\prime}(\Theta)=0$ et $12 \alpha_{b}$ vaut le nombre de points singuliers de la fibre $X_{b}$ du modèle régulier minimal $X$ de $A$;

- $(A ; \lambda)$ est une surface jacobienne; c'est l'objet du $\S 5$ (cf. théorème 5.1 ).

Remarque. La formule de l'énoncé 3.1 précise l'inégalité de Bost suivante (cf. [Bos96]) : avec les mêmes notations, on a la minoration

$$
\hat{\mathrm{h}}\left(A_{\overline{\mathbb{Q}}}\right) \geqslant \frac{2}{N} \sum_{\sigma \in G_{K}} I\left(A_{\sigma} ; \lambda_{\sigma}\right)-\kappa_{0} g .
$$

\section{Préliminaires}

\subsection{Contribution locale}

Soit $A$ une variété abélienne complexe de dimension $g \geqslant 1$. Soit $\lambda: A \rightarrow A^{\vee}$ une polarisation principale de $A$. Notons $\mu$ la mesure de Haar sur $A(\mathbb{C})$ de masse 1 .

Définition. On choisit un faisceau inversible $L$ ample sur $A$ définissant $\lambda$ (on a donc $h^{0}(A ; L)=1$ ), une métrique du cube $\|\cdot\|$ sur $L$, et une section $s \in \Gamma(A ; L)-\{0\}$ (une métrique du cube sur $L$ est par définition une métrique sur $L$ à courbure invariante par translations). On pose

$$
I(A ; \lambda)=-\int_{A(\mathbb{C})} \ln \|s\| \mu+\frac{1}{2} \ln \int_{A(\mathbb{C})}\|s\|^{2} \mu .
$$

On vérifie aisément que ce nombre réel ne dépend pas du choix de $(L ;\|\cdot\| ; s)$. De plus, l'inégalité de convexité de Jensen montre que $I(A ; \lambda)>0$. On vient en fait de définir une fonction continue $I: \mathcal{A}_{g}(\mathbb{C}) \rightarrow \mathbb{R}_{+}$.

Étudions de plus près le cas des courbes elliptiques :

On désigne par $\mathbb{H}_{1}$ le demi-plan supérieur. Pour $\tau \in \mathbb{H}_{1}$, on pose $q=e^{2 i \pi \tau}$ et $\Delta(\tau)=$ $q \prod_{n \geqslant 1}\left(1-q^{n}\right)^{24}$ (rappelons que $\Delta$ est une forme modulaire de poids 12 ).

Soit $E$ une courbe elliptique sur $\mathbb{C}$. On note $\lambda$ la polarisation canonique de $E$. Prenons un $\tau \in \mathbb{H}_{1}$ tel que $E(\mathbb{C}) \simeq \mathbb{C} /(\mathbb{Z}+\tau \mathbb{Z})$.

Proposition 2.1. Avec ces notations, on a la formule suivante :

$$
I(E ; \lambda)=-\frac{1}{24} \ln \left[|\Delta(\tau)|(2 \operatorname{Im} \tau)^{6}\right] .
$$




\section{Hauteur de Faltings et hauteur de Néron-Tate Du Diviseur thêta}

Démonstration. La section neutre $0_{E}$ de $E$ définit un faisceau inversible $L=\mathcal{O}_{E}\left(0_{E}\right)$ et une section globale $1_{L}$ de $L$ (de diviseur $0_{E}$ ). On munit $L$ de la métrique du cube $\|\cdot\|$ telle que $\int_{E(\mathbb{C})}\left\|1_{L}\right\|^{2} \mu=$ $1 / \sqrt{2}$. Notons $g_{0}$ la fonction de Green pour $0_{E}$ normalisée par la condition $\int_{E(\mathbb{C})} g_{0} \mu=0$.

D'après Faltings (cf. lemme p. 417 de [Fal84]), on a la relation suivante :

$$
\forall z \in E(\mathbb{C})-\left\{0_{E}\right\}, \quad g_{0}(z)=-2 \ln \left\|1_{L}\right\|(z)+\frac{1}{12} \ln \left[|\Delta(\tau)|(\operatorname{Im} \tau)^{6}\right] .
$$

On en déduit le résultat en intégrant cette égalité sur $E(\mathbb{C})$ contre $\mu$.

\subsection{Hauteurs}

Lorsque $V$ est une variété de dimension $d$ sur un corps $K$ et $p$ un entier dans $\{0 ; \cdots ; d\}$, on désigne par $\operatorname{Ef}_{p}(V)$ l'ensemble des $p$-cycles effectifs non nuls sur $V$, et par $\operatorname{Ef}(V)$ la réunion $\operatorname{Ef}_{0}(V) \cup \cdots \cup$ $\operatorname{Ef}_{d}(V)$.

Soit $A$ une variété abélienne sur $\overline{\mathbb{Q}}$ de dimension $g \geqslant 1$. Pour tout entier $n \geqslant 2$, notons $[n]: A \rightarrow A$ le morphisme de multiplication par $n$. Soit $L$ un faisceau inversible symétrique et ample sur $A$.

On désigne par $\mathrm{h}_{L}^{\prime}: \operatorname{Ef}(A) \rightarrow \mathbb{R}$ la fonction hauteur de Néron-Tate. Rappelons que c'est la hauteur de Weil pour $L$ caractérisée par la propriété suivante :

$$
\forall n \geqslant 2, \quad \forall E \in \operatorname{Ef}(A), \quad \mathrm{h}_{L}^{\prime}\left([n]_{*} E\right)=n^{2} \mathrm{~h}_{L}^{\prime}(E) .
$$

On sait de plus que $\mathrm{h}_{L}^{\prime}$ est à valeurs positives ou nulles.

Faisons maintenant quelques rappels de la théorie des hauteurs d'Arakelov :

Soit $X$ une variété arithmétique ( $i e$ un schéma intègre, projectif et plat sur $\mathbb{Z}$, tel que la fibre générique $X_{\mathbb{Q}}$ soit régulière) de dimension (absolue) $d$. Pour tout $p \in\{0 ; \cdots ; d\}$, on désigne par $\mathrm{Z}_{p}(X)$ le groupe des $p$-cycles sur $X$.

On note $\widehat{\operatorname{Pic}}(X)$ le groupe des classes d'isométrie de faisceaux inversibles hermitiens sur $X$. Lorsque $\widehat{\mathcal{L}}=(\mathcal{L} ;\|\cdot\|) \in \widehat{\operatorname{Pic}}(X)$, on note $\omega_{\widehat{\mathcal{L}}}$ la $(1 ; 1)$-forme de courbure de $\widehat{\mathcal{L}}$ sur $X(\mathbb{C})$.

Bost, Gillet et Soulé (cf. $\S 3.1$ de [BGS94]) construisent pour tout $p \in\{0 ; \cdots ; d\}$ une application $(p+1)$-linéaire $\widehat{\operatorname{Pic}}(X)^{p} \times \mathrm{Z}_{p}(X) \rightarrow \mathbb{R}$, qui à $\left(\widehat{\mathcal{L}}_{1} ; \cdots ; \widehat{\mathcal{L}}_{p} ; D\right)$ associe la multihauteur $\left\langle\widehat{\mathcal{L}}_{1} \cdots \widehat{\mathcal{L}}_{p} \mid D\right\rangle$ de $D$ relativement à $\left(\widehat{\mathcal{L}}_{1} ; \cdots ; \widehat{\mathcal{L}}_{p}\right)$. Ces applications multilinéaires sont caractérisées par les propriétés suivantes :

- Lorsque $Y$ est un point fermé, on a $\langle\mid Y\rangle=\ln \# k(Y)$;

- Lorsque $Y$ est un fermé intègre de dimension $p \geqslant 1$ et $s$ une section rationnelle non nulle de $\mathcal{L}_{p \mid Y}$, on a

$$
\left\langle\widehat{\mathcal{L}}_{1} \cdots \widehat{\mathcal{L}}_{p} \mid Y\right\rangle=\left\langle\widehat{\mathcal{L}}_{1} \cdots \widehat{\mathcal{L}}_{p-1} \mid \operatorname{div}(s)\right\rangle-\int_{Y(\mathbb{C})} \ln \|s\|_{p} \omega_{\widehat{\mathcal{L}}_{1}} \cdots \omega_{\widehat{\mathcal{L}}_{p-1}}
$$

DÉfinitions. Soient $\widehat{\mathcal{L}} \in \widehat{\operatorname{Pic}}(X)$ et $p \in\{0 ; \cdots ; d\}$. La hauteur d'Arakelov de $D \in \mathrm{Z}_{p}(X)$ relativement à $\widehat{\mathcal{L}}$ est le nombre réel $\mathrm{h}_{\widehat{\mathcal{L}}}(D)=\langle\widehat{\mathcal{L}} \cdots \widehat{\mathcal{L}} \mid D\rangle$.

Lorsque $\mathcal{L}_{\mathbb{Q}}$ est ample sur $X_{\mathbb{Q}}$ et $p \geqslant 1$, la hauteur normalisée de $E \in \operatorname{Ef}_{p-1}\left(X_{\mathbb{Q}}\right)$ relativement à $\widehat{\mathcal{L}}$ est le réel

$$
\mathrm{h}_{\widehat{\mathcal{L}}}^{\prime}(\bar{E})=\frac{\mathrm{h}_{\widehat{\mathcal{L}}}(\bar{E})}{\operatorname{deg}_{\mathcal{L}_{\mathbb{Q}}}(E) p},
$$

où $\bar{E}$ désigne l'adhérence de $E$ dans $X$. 


\section{P. Autissier}

\section{Formule de hauteur}

Lorsque $A$ est une variété abélienne sur $\overline{\mathbb{Q}}$, on note $\hat{\mathrm{h}}(A)$ la hauteur de Faltings de $A$; rappelons sa définition :

On prend une variété abélienne $A^{\prime}$ semi-stable sur un corps de nombres $K \subset \overline{\mathbb{Q}}$ telle que $A_{\overline{\mathbb{Q}}}^{\prime}=A$. On désigne par $X$ le modèle de Néron de $A^{\prime}$ sur $B=\operatorname{Spec}\left(O_{K}\right)$, par $0_{X} \in X(B)$ sa section neutre, et par $\omega_{X}$ le faisceau inversible $\omega_{X}=0_{X}^{*} \Lambda^{g} \Omega_{X / B}$ sur $B$.

On munit $\omega_{X}$ de la métrique $\|\cdot\|_{\mathrm{Fa}}$ telle que pour tout $\sigma \in B(\mathbb{C})=G_{K}$ et tout $\omega \in \Gamma\left(B_{\sigma} ; \omega_{X \sigma}\right)=$ $\Gamma\left(A_{\sigma}^{\prime} ; \Lambda^{g} \Omega_{A_{\sigma}^{\prime} / \mathbb{C}}\right)$, on ait

$$
\|\omega\|_{\mathrm{Fa}}^{2}(\sigma)=\frac{i^{g^{2}}}{2^{g}} \int_{A_{\sigma}^{\prime}(\mathbb{C})} \omega \wedge \bar{\omega}
$$

Alors

$$
\hat{\mathrm{h}}(A)=\frac{\widehat{\operatorname{deg}}\left(\omega_{X} ;\|\cdot\|_{\mathrm{Fa}}\right)}{[K: \mathbb{Q}]}
$$

(cela ne dépend pas du choix de $\left(K ; A^{\prime}\right)$ ).

DÉfinition. Soit $(A ; \lambda)$ une $\overline{\mathbb{Q}}$-variété abélienne de dimension $g \geqslant 1$. On choisit un corps de nombres $K \subset \overline{\mathbb{Q}}$ et une $K$-variété abélienne principalement polarisée $\left(A^{\prime} ; \lambda^{\prime}\right)$ telle que $\left(A_{\overline{\mathbb{Q}}}^{\prime} ; \lambda_{\overline{\mathbb{Q}}}^{\prime}\right)=(A ; \lambda)$. On pose

$$
I^{\prime}(A ; \lambda)=\frac{1}{[K: \mathbb{Q}]} \sum_{\sigma \in G_{K}} I\left(A_{\sigma}^{\prime} ; \lambda_{\sigma}^{\prime}\right) .
$$

Il est facile de voir que ce réel ne dépend pas du choix de $\left(K ; A^{\prime} ; \lambda^{\prime}\right)$.

On montre ici une formule reliant la hauteur de Faltings, la hauteur de Néron-Tate du diviseur thêta, et l'invariant $I$ :

ThÉorème 3.1. Soit $A$ une variété abélienne de dimension $g \geqslant 1$ sur $\overline{\mathbb{Q}}$ ayant partout bonne réduction. Soit $\Theta$ un diviseur effectif symétrique et ample sur $A$, définissant une polarisation principale $\lambda$ de $A$. En posant $L=\mathcal{O}_{A}(\Theta)$, on a alors l'égalité suivante :

$$
\hat{\mathrm{h}}(A)=2 g \mathrm{~h}_{L}^{\prime}(\Theta)+2 I^{\prime}(A ; \lambda)-\kappa_{0} g .
$$

Démonstration. On fixe un corps de nombres $K \subset \overline{\mathbb{Q}}$ de degré $N$, une variété abélienne $A^{\prime}$ sur $K$ et un diviseur effectif $\Theta^{\prime}$ symétrique et ample sur $A^{\prime}$ tels que $\left(A_{\overline{\mathbb{Q}}}^{\prime} ; \Theta_{\overline{\mathbb{Q}}}^{\prime}\right)=(A ; \Theta)$.

Désignons par $f: X \rightarrow B$ le modèle de Néron de $A^{\prime}$ sur $B=\operatorname{Spec}\left(O_{K}\right)$ et par $T$ l'adhérence de $\Theta^{\prime}$ dans $X$. Le diviseur $T$ définit un faisceau inversible $\mathcal{L}_{0}=\mathcal{O}_{X}(T)$ (symétrique et ample) et une section globale $1_{T}$ de $\mathcal{L}_{0}$.

Pour tout $\sigma \in G_{K}$, notons $\mu_{\sigma}$ la mesure de Haar sur $A_{\sigma}^{\prime}(\mathbb{C})$ de masse 1 . Munissons $\mathcal{L}_{0}$ de la métrique du cube $\|\cdot\|_{0}$ vérifiant $\int_{X_{\sigma}(\mathbb{C})}\left\|1_{T}\right\|_{0}^{2} \mu_{\sigma}=2^{-g / 2}$ pour tout $\sigma \in G_{K}$. On pose $\widehat{\mathcal{L}}_{0}=\left(\mathcal{L}_{0} ;\|\cdot\|_{0}\right)$ et $\widehat{\omega}_{X}=\left(\omega_{X} ; c\|\cdot\|_{\mathrm{Fa}}\right)$ où $c$ est une constante qui sera choisie dans un instant.

En notant $\widehat{\mathcal{L}}_{1}=\widehat{\mathcal{L}}_{0}^{\otimes 8} \otimes f^{*} \widehat{\omega}_{X}^{\otimes-4}$ et $\widehat{\mathcal{O}}_{B}=\left(\mathcal{O}_{B} ;|\cdot|\right)$, la 'formule clef' de Moret-Bailly (cf. théorèmes 0.2 et 3.3 de [Mor90]) produit un isomorphisme $0_{X}^{*} \mathcal{L}_{1} \simeq \mathcal{O}_{B}$ qui est une isométrie $0_{X}^{*} \widehat{\mathcal{L}}_{1} \simeq \widehat{\mathcal{O}}_{B}$ lorsque $c=(2 \pi)^{-g}$.

Par le théorème du cube, on en déduit naturellement une isométrie $[n]^{*} \widehat{\mathcal{L}}_{1} \simeq \widehat{\mathcal{L}}_{1}^{\otimes n^{2}}$ sur $X$ pour chaque entier $n \geqslant 2$, où $[n]$ désigne la multiplication par $n$ sur le $O_{K}$-schéma abélien $X$.

La formule de projection (cf. proposition 3.2.1(iii) de [BGS94]) donne $\mathrm{h}_{\widehat{\mathcal{L}}_{1}}^{\prime}\left([n]_{*} \bar{E}\right)=\mathrm{h}_{[n]^{*} \widehat{\mathcal{L}}_{1}}^{\prime}(\bar{E})=$ $n^{2} \mathrm{~h}_{\widehat{\mathcal{L}}_{1}}^{\prime}(\bar{E})$ pour tout $E \in \operatorname{Ef}\left(A^{\prime}\right)$ et tout $n \geqslant 2$. 


\section{Hauteur de Faltings et hauteur de Néron-Tate Du Diviseur thêta}

On en déduit que la hauteur d'Arakelov coïncide avec la hauteur de Néron-Tate, ie que $\mathrm{h}_{\widehat{\mathcal{L}}_{1}}^{\prime}(\bar{E})=\mathrm{h}_{L \otimes 8}^{\prime}\left(E_{\overline{\mathbb{Q}}}\right)=8 \mathrm{~h}_{L}^{\prime}\left(E_{\overline{\mathbb{Q}}}\right)$ pour tout $E \in \operatorname{Ef}\left(A^{\prime}\right)$. On a en particulier $\mathrm{h}_{\widehat{\mathcal{L}}_{1}}(X)=0$ et $\mathrm{h}_{\widehat{\mathcal{L}}_{1}}(T)=g ! 8^{g} g N \mathrm{~h}_{L}^{\prime}(\Theta)$ (observons que $\operatorname{deg}_{L}(\Theta)=g !$ ).

FAIT. Pour tout $p \in\{0 ; \cdots ; g+1\}$ et tout $D \in \mathrm{Z}_{p}(X)$, on a la relation suivante :

$$
\frac{1}{8^{p}} \mathrm{~h}_{\widehat{\mathcal{L}}_{1}}(D)=\mathrm{h}_{\widehat{\mathcal{L}}_{0}}(D)-\operatorname{deg}_{L}\left(D_{\overline{\mathbb{Q}}}\right) \frac{p N}{2}[\hat{\mathrm{h}}(A)-\ln c] .
$$

Prouvons ce Fait. En utilisant la multilinéarité, on obtient

$$
\frac{1}{8^{p}} \mathrm{~h}_{\widehat{\mathcal{L}}_{1}}(D)=\sum_{j=0}^{p} \mathrm{C}_{p}^{j}(-2)^{-j}\left\langle\widehat{\mathcal{L}}_{0}^{p-j}\left(f^{*} \widehat{\omega}_{X}\right)^{j} \mid D\right\rangle
$$

Puisque $\widehat{\omega}_{X}$ vit sur $B$ qui est de dimension 1, on a (cf. proposition 2.3.1(iii) de [BGS94, p. 938]) l'égalité $\left\langle\widehat{\mathcal{L}}_{0}^{p-j}\left(f^{*} \widehat{\omega}_{X}\right)^{j} \mid D\right\rangle=0$ pour tout $j \geqslant 2$, ainsi que $\left\langle\widehat{\mathcal{L}}_{0}^{p-1} f^{*} \widehat{\omega}_{X} \mid D\right\rangle=\operatorname{deg}_{L}\left(D_{\overline{\mathbb{Q}}}\right) N[\hat{\mathrm{h}}(A)-\ln c]$. D'où le fait énoncé.

Maintenant, on écrit la relation (1) pour $D=T$ et pour $D=X$, puis on soustrait membre à membre; on trouve alors

$$
\frac{1}{8^{g}} \mathrm{~h}_{\widehat{\mathcal{L}}_{1}}(T)-\frac{1}{8^{g+1}} \mathrm{~h}_{\widehat{\mathcal{L}}_{1}}(X)=\mathrm{h}_{\widehat{\mathcal{L}}_{0}}(T)-\mathrm{h}_{\widehat{\mathcal{L}}_{0}}(X)+g ! \frac{N}{2}[\hat{\mathrm{h}}(A)-\ln c] .
$$

Par ailleurs, grâce à la propriété $(\ddagger)$ des hauteurs d'Arakelov, on a

$$
\mathrm{h}_{\widehat{\mathcal{L}}_{0}}(T)-\mathrm{h}_{\widehat{\mathcal{L}}_{0}}(X)=\sum_{\sigma \in G_{K}} \int_{X_{\sigma}(\mathbb{C})}\left(\ln \left\|1_{T}\right\|_{0}\right) g ! \mu_{\sigma}=-g ! N\left[I^{\prime}(A ; \lambda)+\frac{g}{4} \ln 2\right] .
$$

On obtient donc l'égalité

$$
g ! g N \mathrm{~h}_{L}^{\prime}(\Theta)=-g ! N\left[I^{\prime}(A ; \lambda)+\frac{g}{4} \ln 2\right]+g ! \frac{N}{2}[\hat{\mathrm{h}}(A)-\ln c] .
$$

On conclut en divisant par $g ! N / 2$.

Remarquons que cette formule précise l'inégalité de Bost suivante (cf. théorème $\S 3$ de [Bos96]). ThÉorème (Bost). Soit $(A ; \lambda)$ une $\overline{\mathbb{Q}}$-variété abélienne de dimension $g \geqslant 1$, principalement polarisée. On a alors la minoration $\hat{\mathrm{h}}(A) \geqslant 2 I^{\prime}(A ; \lambda)-\kappa_{0} g$.

\section{4. Étude asymptotique}

Soit $g$ un entier $\geqslant 1$. On note $\mathbb{H}_{g}$ l'espace de Siegel des matrices $\Omega \in \mathrm{M}_{g}(\mathbb{C})$ symétriques telles que $\operatorname{Im} \Omega$ soit définie positive. À tout $\Omega \in \mathbb{H}_{g}$ on associe la fonction thêta définie par

$$
\forall z \in \mathbb{C}^{g}, \quad \theta_{\Omega}(z)=\sum_{m \in \mathbb{Z}^{g}} \exp \left(i \pi^{\mathrm{t}} m \Omega m+2 i \pi^{\mathrm{t}} m z\right) .
$$

Soit $(A ; \lambda)$ une $\mathbb{C}$-variété abélienne de dimension $g$, principalement polarisée. Choisissons un $\Omega \in \mathbb{H}_{g}$ tel que $A(\mathbb{C}) \simeq \mathbb{C}^{g} /\left(\mathbb{Z}^{g}+\Omega \mathbb{Z}^{g}\right)$, que $\lambda$ soit induite par $\Theta=\operatorname{div}\left(\theta_{\Omega}\right)$, et que $Y=\operatorname{Im} \Omega$ soit réduite au sens de Minkowski (cf. $\S$ V.4 de $[\operatorname{Igu} 72]$ ).

En notant $a_{1} ; \cdots ; a_{g}$ les coefficients diagonaux de $Y$, on a en particulier $a_{k} \geqslant \sqrt{3} / 2$ pour tout $k \in\{1 ; \cdots ; g\}$.

Proposition 4.1. Il existe deux constantes $\varepsilon_{g}>0$ et $c_{g}>0$ ne dépendant que de $g$ telles que $I(A ; \lambda) \geqslant \varepsilon_{g} \operatorname{Tr}(Y)-c_{g}$. 


\section{P. Autissier}

Démonstration. On désigne par $\mu$ la mesure de Haar sur $A(\mathbb{C})$ et par $\nu$ la mesure de Lebesgue sur $\mathbb{R}^{g}$. On pose $L=\mathcal{O}_{A}(\Theta)$ et on note $\theta$ la section globale de $L$ définie par $\Theta$. On munit $L$ de la métrique $\|\cdot\|$ définie par

$$
\forall z=x+i y \in \mathbb{C}^{g}, \quad\|\theta\|(z)=\sqrt[4]{\operatorname{det}(Y)} \exp \left(-\pi^{\mathrm{t}} y Y^{-1} y\right)\left|\theta_{\Omega}(z)\right| .
$$

C'est une métrique du cube sur $L$ (cf. $\S 3$ de [Mor90]) et on a

$$
\ln \int_{A(\mathbb{C})}\|\theta\|^{2} \mu=-\frac{g}{2} \ln 2 .
$$

Maintenant, majorons le terme $\int_{A(\mathbb{C})} \ln \|\theta\| \mu$. Posons $F=\left[-\frac{1}{2} ; \frac{1}{2}\right]^{g}$. En utilisant l'inégalité de Jensen puis la formule de Parseval, on obtient pour tout $y \in \mathbb{R}^{g}$ :

$$
\begin{aligned}
\int_{F} \ln \|\theta\|^{2}(x+\Omega y) \nu(x) & \leqslant \ln \int_{F}\|\theta\|^{2}(x+\Omega y) \nu(x) \\
& =\ln \left[\sqrt{\operatorname{det}(Y)} \sum_{m \in \mathbb{Z}^{g}} \exp \left[-2 \pi^{\mathrm{t}}(m+y) Y(m+y)\right] .\right.
\end{aligned}
$$

On pose $D=\operatorname{Diag}\left(a_{1} ; \cdots ; a_{g}\right)$. D'après la théorie de la réduction (cf. corollaire 2 de $[\operatorname{Igu} 72$, p. 193]), il existe $c>0$ ne dépendant que de $g$ tel que ${ }^{t} y Y y \geqslant c^{t} y D y$ pour tout $y \in \mathbb{R}^{g}$. On en déduit

$$
\begin{aligned}
2 \int_{A(\mathbb{C})} \ln \|\theta\| \mu-\frac{1}{2} \ln [\operatorname{det}(Y)] & \leqslant \int_{F} \ln \left[\sum_{m \in \mathbb{Z}^{g}} \exp \left[-2 c \pi^{\mathrm{t}}(m+y) D(m+y)\right]\right] \nu(y) \\
& =\sum_{k=1}^{g} \int_{-1 / 2}^{1 / 2} \ln \left[\sum_{m \in \mathbb{Z}} e^{-2 c \pi a_{k}(m+y)^{2}}\right] d y .
\end{aligned}
$$

On conclut en appliquant $g$ fois le lemme 4.2 ci-dessous et en remarquant que

$$
\frac{1}{g} \ln [\operatorname{det}(Y)] \leqslant \ln \left[\frac{1}{g} \operatorname{Tr}(Y)\right] \leqslant \frac{c}{g} \operatorname{Tr}(Y)-1-\ln c .
$$

Lemme 4.2. Soit $a>0$. On a la majoration suivante :

$$
\int_{-1 / 2}^{1 / 2} \ln \left[\sum_{m \in \mathbb{Z}} e^{-a(m+y)^{2}}\right] d y \leqslant-\frac{a}{12}+\ln \left(1+\frac{1}{a}\right) .
$$

Démonstration. L'inégalité de Jensen donne

$$
\int_{-1 / 2}^{1 / 2} \ln \left(\sum_{m \in \mathbb{Z}} e^{-a m^{2}-2 a m y}\right) d y \leqslant \ln \int_{-1 / 2}^{1 / 2}\left(\sum_{m \in \mathbb{Z}} e^{-a m^{2}-2 a m y}\right) d y .
$$

Un calcul montre que le membre de droite vaut $\ln \left[1+1 / a-\sum_{m \geqslant 1}\left(e^{-a m(m+1)} / a m(m+1)\right)\right]$. D'où le résultat.

Remarque. La proposition 4.1 implique que $I(A ; \lambda)$ tend vers $+\infty$ lorsque $(A ; \lambda)$ tend vers l'infini dans $\mathcal{A}_{g}(\mathbb{C})$. Autrement dit, pour tout $Q \in \mathbb{R}$, l'ensemble $\left\{(A ; \lambda) \in \mathcal{A}_{g}(\mathbb{C}) \mid I(A ; \lambda) \leqslant Q\right\}$ est compact.

\section{Surfaces jacobiennes}

Soit $K$ un corps de nombres de degré $N$. Soit $f: X \rightarrow B$ une surface arithmétique régulière semi-stable sur $B=\operatorname{Spec}\left(O_{K}\right)$, telle que la fibre générique $C=X_{K}$ soit de genre 2 . 


\section{Hauteur de Faltings et hauteur de Néron-Tate Du Diviseur thêta}

Soit $M$ un faisceau inversible (de degré 1$)$ sur $C$ tel que $M^{\otimes 2}=\Omega_{C / K}$. Désignons par $(J ; \lambda)$ la jacobienne de $C$. Le faisceau $M$ définit un plongement $C \hookrightarrow J$, dont on note $\Theta$ l'image. On pose $L=\mathcal{O}_{J}(\Theta)$.

ThÉorème 5.1. Avec ces notations, on a la formule

$$
\hat{\mathrm{h}}\left(J_{\overline{\mathbb{Q}}}\right)=4 \mathrm{~h}_{L}^{\prime}(\Theta)+\frac{1}{N} \sum_{b \in B} \alpha_{b} \ln \mathrm{N}_{b}+\frac{2}{N} \sum_{\sigma \in G_{K}} I\left(J_{\sigma} ; \lambda_{\sigma}\right)-2 \kappa_{0},
$$

où pour tout point fermé $b \in B, \alpha_{b}$ est un rationnel positif ou nul calculable en fonction du graphe de réduction de la fibre $X_{b}$ au-dessus de $b$.

Démonstration. On note $\widehat{\mathcal{K}}$ le faisceau canonique sur $X$, muni des métriques d'Arakelov.

Pour tout $\sigma \in G_{K}$, on désigne par $\delta_{\sigma}$ l'invariant de Faltings de $C_{\sigma}(\mathbb{C})$ (cf. [Fal84, p. 402]). Pour chaque point fermé $b \in B$, on note $\delta_{b}$ le nombre géométrique de points singuliers de la fibre $X_{b}$. La formule de Noether arithmétique de Moret-Bailly (cf. théorème 2.5 de [Mor89]) donne

$$
6 \hat{\mathrm{h}}\left(J_{\overline{\mathbb{Q}}}\right)=\frac{\langle\widehat{\mathcal{K}} \cdot \widehat{\mathcal{K}}\rangle}{2 N}+\frac{1}{2 N} \sum_{b} \delta_{b} \ln \mathrm{N}_{b}+\frac{1}{2 N} \sum_{\sigma} \delta_{\sigma}-4 \ln (2 \pi) .
$$

Par ailleurs, d'après Zhang [Zha93, théorème 5.5] et Abbes [Abb97, théorème 5.2 et remarque p. 161], on a

$$
\frac{\langle\widehat{\mathcal{K}} . \widehat{\mathcal{K}}\rangle}{2 N}=4 \mathrm{~h}_{L}^{\prime}(\Theta)+\frac{1}{2 N} \sum_{b} e_{b} \ln \mathrm{N}_{b}
$$

où les $e_{b}$ sont des rationnels positifs ou nuls explicites (l'hypothèse $g=2$ est ici cruciale).

Désignons par $\Delta \in \Gamma\left(B ;\left(\Lambda^{2} f_{*} \mathcal{K}\right){ }^{\otimes 10}\right)$ le discriminant de $C$ (cf. $\S 2$ de [Uen88]). Pour tout point fermé $b \in B$, notons $d_{b}$ l'ordre d'annulation de $\Delta$ en $b$. Par la proposition 4 de [Bos87], on a $\ln \|\Delta\|(\sigma)=4 I\left(J_{\sigma} ; \lambda_{\sigma}\right)-\delta_{\sigma}+2 \ln \left(8 \pi^{2}\right)$ pour tout $\sigma \in G_{K}$. On en déduit

$$
\frac{1}{2 N} \sum_{b} d_{b} \ln \mathrm{N}_{b}+\frac{1}{2 N} \sum_{\sigma}\left(\delta_{\sigma}-4 I\left(J_{\sigma} ; \lambda_{\sigma}\right)\right)-\ln \left(8 \pi^{2}\right)=5 \hat{\mathrm{h}}\left(J_{\overline{\mathbb{Q}}}\right) .
$$

En additionnant membre à membre les égalités (2), (3) et (4), on obtient le résultat avec $\alpha_{b}=$ $\left(\delta_{b}+e_{b}-d_{b}\right) / 2$.

Remarque. On peut expliciter $\alpha_{b}$. En reprenant la classification exposée dans la table 1 de [Mor96], on trouve:

- Cas I (bonne réduction): $\alpha_{b}=0$;

- $\operatorname{Cas} \operatorname{II}(x): \alpha_{b}=0$;

- $\operatorname{Cas} \operatorname{III}(x): \alpha_{b}=x / 12$;

- $\operatorname{Cas} \operatorname{IV}(x ; y): \alpha_{b}=y / 12$;

- Cas $\mathrm{V}(x ; y): \alpha_{b}=(x+y) / 12$;

- $\mathrm{Cas} \operatorname{VI}(x ; y ; z): \alpha_{b}=(y+z) / 12$;

- $\operatorname{Cas} \operatorname{VII}(x ; y ; z): \alpha_{b}=[x y z /(x y+y z+z x)+x+y+z] / 12$.

\section{REMERCIEMENTS}

Je remercie Jean-Benoît Bost pour m'avoir exposé sa démonstration de l'inégalité (*). Je remercie également Marc Hindry et Nicolas Ratazzi pour d'intéressantes discussions concernant cet article. Enfin, je remercie le rapporteur pour ses suggestions avisées. 


\section{Hauteur de Faltings et hauteur de Néron-Tate du Diviseur thêta}

\section{BIBLIOGRAPHIE}

Abb97 A. Abbes, Hauteurs et discrétude, Astérisque 245 (1997), 141-166.

Bos87 J. B. Bost, Fonctions de Green-Arakelov, fonctions thêta et courbes de genre 2, C. R. Acad. Sci. Paris Sér. I Math. 305 (1987), 643-646.

Bos96 J. B. Bost, Arakelov geometry of abelian varieties, Preprint 51 (1996), Max-Planck-Institut für Mathematik.

BGS94 J. B. Bost, H. Gillet and C. Soulé, Heights of projective varieties and positive Green forms, J. Amer. Math. Soc. 7 (1994), 903-1027.

Fal84 G. Faltings, Calculus on arithmetic surfaces, Ann. of Math. (2) 119 (1984), 387-424.

Igu72 J. Igusa, Theta functions, Grundlehren der Mathematischen Wissenschaften, vol. 194 (Springer, New York, 1972).

Mor85 L. Moret-Bailly, Pinceaux de variétés abéliennes, Astérisque 129 (1985).

Mor89 L. Moret-Bailly, La formule de Noether pour les surfaces arithmétiques, Invent. Math. 98 (1989), 491-498.

Mor90 L. Moret-Bailly, Sur l'équation fonctionnelle de la fonction thêta de Riemann, Compositio Math. 75 (1990), 203-217.

Mor96 A. Moriwaki, Bogomolov conjecture for curves of genus 2 over function fields, J. Math. Kyoto Univ. 36 (1996), 687-695.

Uen88 K. Ueno, Discriminants of curves of genus 2 and arithmetic surfaces, Algebr. Geom. Commutative Algebra 2 (1988), 749-770.

Zha93 S. Zhang, Admissible pairing on a curve, Invent. Math. 112 (1993), 171-193.

Pascal Autissier pascal.autissier@univ-rennes1.fr

IRMAR, Université de Rennes I, Campus de Beaulieu, 35042 Rennes cedex, France 